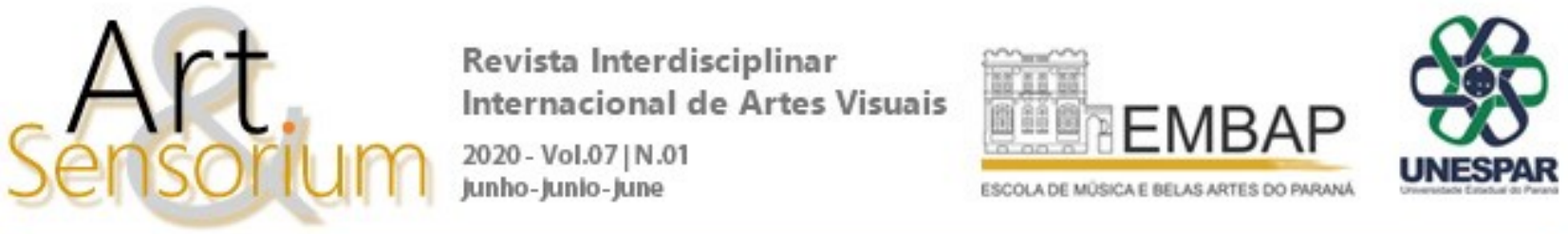

\title{
EXPONER LA INVESTIGACIÓN ARTÍSTICA EN MUSEOS Y GALERÍAS
}

\author{
DOI: https://doi.org/10.33871/23580437.2020.7.1.99-118
}

Ana Tirado de la Chica $^{1}$

\begin{abstract}
Resumen: Este trabajo aborda la musealización de la investigación artística en la exposición de arte. Se realiza un estudio comparado de dos casos internacionales de exposición, de acuerdo a cuestiones de la investigación artística: Usterile Clinic (2016) de Aida Silvestri, en Autograph ABP-Rivington Place (Londres, Reino Unido); e Itinerarios del vértigo (2015) de Sandra Silva, en el Museo de Arte de Pereira (Pereira, Colombia). Los resultados aportan información sobre los contenidos que se encuentran en sala para una investigación artística, y sobre las formas museológicas y museográficas en las que estos se han articulado en las exposiciones. Las conclusiones están dirigidas a introducir en la exposición de arte aquellos aspectos involucradose en la producción artística.
\end{abstract}

Palabras clave: Arte; Museología; Investigación artística; Exposición

\section{EXHIBIT THE ARTISTIC RESEARCH IN MUSEUMS AND GALLERIES}

Abstract: This paper deals with the musealization of artitic research in art exhibitions. The methodology is based on a comparative study of two international cases of art exhibitions, regarding to variables of artistic research as theoreical framework. The two cases of the sample are: Unsterile Clinic (2016) by Aida Silvestri, in Autograph ABP-Rivington Place, (London, United Kingdom); and Itinerarios del vértigo (2015) by Sandra Silva, at the Art Museum of Pereira (Pereira, Colombia). Results are about contents and forms of musealization of artistic research adopted in both art exhibitions. Conclusiones are directed to introduce in art exhibitions those aspects involved in art production.

Keywords: Art, Museology; Museology; Artistic research; Exhibition

\footnotetext{
${ }^{1}$ https://orcid.org/0000-0001-5761-7288. Licenciada en Historia del Arte (2010) y Doctora en Patrimonio (2014) por la Universidad de Jaén (España), ocupa el puesto de Docente e Investigadora del Área de Didáctica de la Expresión Plástica desde el año 2013. Dedica su investigación al estudio museológico y cultural de los museos y exposiciones de arte. Ha realizado sendas estancias por varios países que le han permitido adquirir una mirada internacional de su investigación: Centre d'Études sur l'Actuel et le Quotidien-Université Paris Descartes (2012-2013), Departamento de Artes Visuales-Universidad del Valle (2015), Educational Studies Department-Goldsmiths-University of London (2016). Universidad de Jaén - Jaén (España).atirado@ujaen.es
} 


\section{Introducción}

La forma de exponer las obras es una cuestión de interés constante en el ámbito de la museología del arte. Museos y galerías de arte han experimentado en los últimos años un importante desarrollo de sus servicios culturales y de exposiciones. Respecto de la digitalización de la información (MARTÍN, 2015), con el desarrollo de dispositivos electrónicos de información, como audio-guías, aplicaciones digitales para smartphone, iPads, etc. Respecto de la virtualización de los contenidos artísticos, como catálogo en línea de las colecciones, documentación audiovisual de conferencias, reportajes, etc. Y respecto de la museografía, por ejemplo, con la inclusión en sala de señalética de rótulos con preguntas (LORENTE, 2015, 2013). Así, como decía Dubé, entendemos que "[...] las maneras de exponer son tributarias de las maneras de pensar la exposición, [dado que] la ciencia, por su rigurosos método de examen empírico, va a influir enormemente en los modos de exposición museística gracias a su enfoque constantemente renovador de la materia estudiada" (DUBÉ, 1995, p. 5).

Dos casos recientes de exposición suscitan una nueva forma de pensar la exposición de arte: exponer la investigación artística, y no solo las obras del/a artista. Se tratan de la exposición Unsterile clinic $^{2}$, en Rivington Place (Londres, Reino Unido), del 8 de julio al 17 de septiembre de 2016, con obra monográfica de la artista Aida Silvestri; y la exposición Itinerarios del vértigo ${ }^{3}$, en el Museo de Arte de Pereira (Pereira, Colombia), del 26 de junio al 16 de agosto de 2015, con obra monográfica de la artista Sandra Silva. Ambos casos tienen en común que incluyeron en sala otros dispositivos y artefactos con contenidos alusivos al proceso de producción artística, además de las obras. Esta coincidencia resulta de especial interés, porque que se encuentra en dos casos de exposición recientes y actuales, pero que están muy separados geográficamente entre sí (Londres y Pereira), y donde uno es totalmente independiente al otro.

Por tanto, el presente trabajo se interroga por las formas de musealización de la investigación artística adoptadas en estos dos casos interancionales de exposición de arte: Unsterile clinic (2016) en el Rivington Place (Londres, Reino Unido), con obra de la artista Aida Silvestri; e Itinerarios del vértigo (2015) en el Museo de Arte de Pereira (Pereira, Colombia), con obra de la artista Sandra Silva. Así, el conjunto de este trabajo se aborda en tres apartados: en primer lugar, una revisión teórica del tema, sobre el cambio museológico entre entender la exposición de arte con un carácter exclusivamente estético, y que tradicionalmente ha sido dominante, y la exposición de acuerdo a contenidos artísticos; en segundo lugar se describe el método empleado y la muestra de estudio; finalmente, se aborda la discusión de los resultados según contenidos de investigación artística y según las formas que estos adquieren en su musealización en la exposición.

\section{De la exposición estética del arte a la exposición de contenidos artísticos}

La forma de exponer el arte ha estado dominada por la tipología estética de la exposición (LORENTE, 2008; RICO, 2007; ÁVILA, 2003; ALONSO, 2001; HOOPER-GREENHILL, 1992). En este sentido, Alonso distingue cuatro tipologías de exposición, según las siguientes: la simbólica, la comercial, la

\footnotetext{
${ }^{2}$ Página web dedica a la exposición Unsterile clinic en la web de la artista Aida Silvestri: https://www.aidasilvestri.com/section787655.html

${ }^{3}$ MUSEO DE ARTE DE PEREIRA. (18/07/2015). Sandra Silva: Itinerarios del vértigo [archivo de vídeo]. Disponible en: https://www.youtube.com/watch?v=evrv32F-_Xk [Consultado: 9/04/2020].
}

R. Inter. Interdisc. Art\&Sensorium, Curitiba, v.7, n.1, p. 099 - 118 Jan.- Jun. 2020 
documental y la estética (ALONSO, 2001, p. 202). De entre estas cuatro, la que se refiere a la exposición del arte es la última, la exposición estética. Siguiendo a Alonso (2001), el tipo de exposición estética se refiere al valor artístico de las obras y está asociado a los museos y galerías de arte, mientras que el tipo documental, que se refire al valor informativo o científico de los objetos, está asociado a museos de carácter científico o técnico, como los ecomuseos, por ejemplo.

La tipología estética de la exposición de arte es heredera del legado históriográfico de la historia del arte de Winckelmann $(1964,1763,1987)$, anteriormente focalizada sobre los artistas y sus planteamientos de producción del arte y, desde entonces, dirigida hacia las cualidades estéticas de las obras por sí mismas. En nuestra época contemporánea, se habla de la "exposición creativa" (PÉREZ, 2007), en la que se explotan las cualidades escenográficas y sensitivas de la exposición, interviniendo sobre muros, techos, etc., con elementos pictórico-gráficos para intensificar la experiencia estética de las obras en sala. Una de las apuestas más arriesgadas en este sentido la constituye el Palais de Tokyo 4 (París), inaugurado en 2002 y fundado bajo las premisas teóricas del arte de Nicolas Bourriaud sobre la Estética Relacional (BOURRIAUD, 2001) y la Postproducción (BOURRIAUD, 2003). El Palais de Tokyo está emplazado en el antiguo pabellón del museo de arte moderno de la ciudad de París de la Exposición Internacional de 1937. Se distingue por dejar en bruto la estructura de construcción, sin ninguna intervención de embellecimiento; así, su aspecto interior es de hormigón visto, como también todo el sistema de cableado y tuberías. Al visitarlo, resulta de un espacio no pre-definido, de modo que no existen riesgos de "dañar" el edificio (manchar, desconchar, rotura, etc.). Este planteamiento del espacio lo dispone para la más variadas propuestas de creación, como Acquaalta en 2015 de Céleste Boursier-Mougenot (PALAIS DE TOKYO, 2015), que consistía en instalaciones audiovisuales en la base de un estanque de agua que se surcaba en pequeñas barcas.

(...) pensar que las obras proponen escenarios y que el arte es una forma de uso del mundo, una negociación infinita entre puntos de vista. Somos nosotros, observadores, quienes ponemos en evidencia estas relaciones. Somos nosotros quienes juzgamos las obras de arte en función de las relaciones que ellas producen en el interior del contexto específico en el que ellas se debaten. Porque el arte, y finalmente no conozco otra definición que las englobe todas, es una actividades que consiste en producir relaciones con el mundo, en materializar en una forma $u$ otra sus relaciones con el espacio y el tiempo (BOURRIAUD, 2003, pp. 92-935).

Los ejemplos para una actualización y experimentación en las formas de exponer el arte contempráneo y actual pueden remontarse hasta los años sesenta del siglo pasado. En esta época la Documenta 8 de 1967, en Kassel (Alemania), que estuvo dirigidad por Manfred Schneckenburger, protagonizó un ejemplo paradigmático al incluir en el proyecto curatorial una amplia variedad de medios, soportes y formatos artísticos del momento (vídeo, instalaciones, performances, etc.). Así, se pretendía fracturar la dominancia de la pintura. En palabras de Guash: "los nuevos medios, la arquitectura, el vídeo, las instalaciones, las performances, el diseño, con todo lo que ello significaba de carpetazo al ya "anquilosado" soporte pictórico" (GUASH, 1996, p. 152). La propuesta museológica del Centro Pompidou en 1977 también contituyó otro ejemplo paradigmático en este sentido, aunque pronto se reveló como un formato de grandes exposiciones, que poco o nada dejaba a la innovación artística además de un "proto inevitable de hipermercado cultural" (ALONSO, 2001, p. 86). Otro ejemplo de referencia internacional ha sido la nueva ordenación de la Tate Modern de Londres, que se hizo en el año 2000.

\footnotetext{
${ }^{4}$ Página web oficial del Palais Tokyo: https://www.palaisdetokyo.com/

5 Traducción de la autora del original en francés.

R. Inter. Interdisc. Art\&Sensorium, Curitiba, v.7, n.1, p. 099 - 118 Jan.- Jun. 2020
} 
Esta estuvo basada en una ordenación de obras de la colección basada en categorías de género y afinidades formales, según "diálogos visuales no ordenados diacrónicamente" (LORENTE, 2015, pp. 115-116).

\section{Método}

Para conocer cómo se articula esta transferencia del conocimiento artístico en la exposición de arte, se adopta un método de estudio comparado de casos (MASSERONI, 2010), según una muestra de dos casos internacionales de exposición, que son representativos de integrar dispositivos en sala alusivos a la producción artística de la obra. De este modo, el análisis del estudio está dirigido a conocer cuáles son los contenidos que estos dispositivos introducen en sala y si estos suponen una aportación al carácter científico de la exposición de arte. Se definen dos variables para el análisis, según los dispositivos museográficos internos en sala y según los contenidos de estos mismos dispositivos. Debido a la variedad tipológica de los dispositivos en sala entre los casos y a que constituyen una elaboración original de cada montaje expositivo, el análisis del estudio se aborda de forma deductiva. Para delimitar los dispostivos que se analizan se establece que son dispositivos de estudio los elementos museográficos internos en sala de las exposiciones, que contengan información relativa a las obras, sus autores, historia y producción artística, sin discriminar por tipología del formato (señalética, vídeo, gráfico...) ni lenguaje empleado (escrito, visual, etc.).

\section{La muestra}

La muestra está compuesta por dos casos internacionales de exposición de arte, que son:

- $\quad$ Unsterile clinic (2016) en el Rivington Place, en Londres, con obra de la artista Aida Silvestri.

- Itinerarios del vértigo (2015) en el Museo de Arte de Pereira, en Pereira (Colombia), con obra de la artista Sandra Silva.

El ámbito internacional de la muestra evidencia que la cuestión de la musealización del arte y de qué formas actuales adquiere la exposición artística es una cuestión global y común a todo el ámbito, de modo que no está ligada a focos concretos de debate. Por otro lado, ambas exposiciones de arte de la muestra comparten una temporalidad muy próxima, con apenas un año de distancia temporal: una corresponde con el año 2015 y otra con 2016. Esta periodicidad de la muestra evidencia la actualidad del tema de estudio, y de la que se espera obtener evidencias para contribuir al futuro desarrollo de la cuestión.

\section{Exposición Unsterile Clinic en el Autrograph ABP-Rivington Place de Londres (Reino Unido).}

Unsterile clinic fue una exposición de arte con obra monográfica de la artista Aida Silvestri ${ }^{6}$, que estuvo expuesta en la galería de fotografía Autrograph $\mathrm{ABP}^{7}$, con sede en el centro del Rivington Place,

\footnotetext{
${ }^{6}$ Página web dedica a la exposición Unsterile clinic en la web de la artista Aida Silvestri: https://www.aidasilvestri.com/section787655.html

${ }^{7}$ Autograph ABP es una galería de arte afincada en la ciudad de Londres dedica a obra de fotografía y de video y a temas de identificación, corporeidad y justicia social. Esta galería destaca en Londres por su dedicación al trabajos de artistas de
}

R. Inter. Interdisc. Art\&Sensorium, Curitiba, v.7, n.1, p. 099 - 118 Jan.- Jun. 2020 
(Londres, Reino Unido), del 8 de julio al 17 de septiembre de 2016. La muestra de la exposición y de la obras versaban sobre el tema de la Mutilación Genital Femenina (MGF, en adelante). Era resultado de un proyecto artístico que la artista había realizado sobre la MGF durante los años 2015 y 2016, y que estuvo subvenciado por el organismo del "Arts Council England".

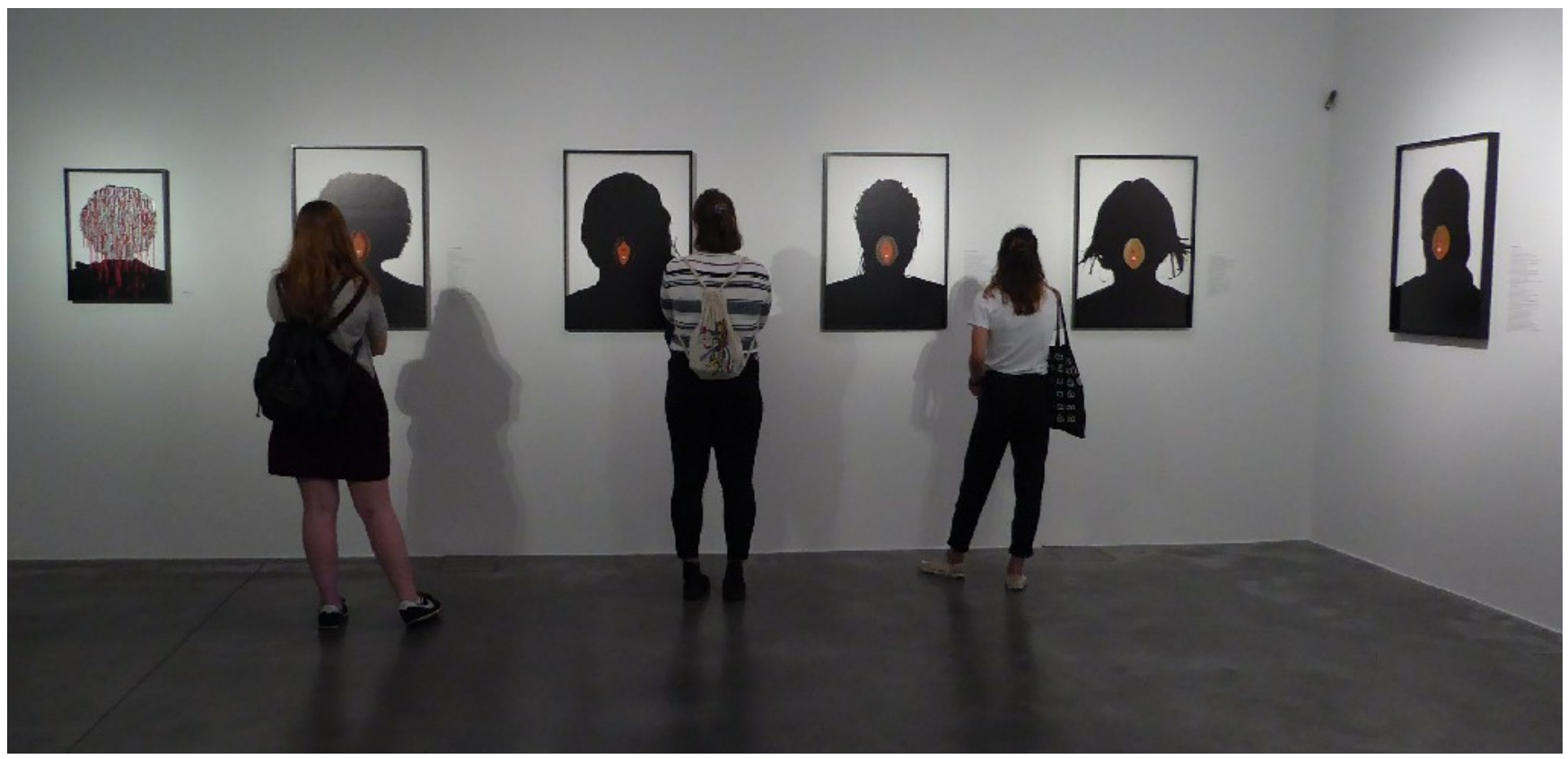

Imagen 1. Interior de la exposición Unsterile Clinic. 2016. Aida Silvestri. Galería Autograph ABPRivington Place, Londres. Imagen propia, 2016.

Como se aprecia en la Imagen 1, la obra de Aida Silvestri en la exposición de Unsterile Clinic era de tipo visual. Estaba realizada en técnica mixta: sobre una primera fotografía impresa en blanco y negro con la silueta del retrato de una mujer, se habían añadido otra serie de objetos, pequeños, y a color. Estos objetos estaban colocados en la zona de la boca del retrato, y representaban una vulva de mujer mutilada. De esta forma, cada retrato representaba un tipo de MGF, y según la artista había podido también identificar en su investigación. La representación de los labios mayores y menores estaba realizada con material de cuerpo en colores marrones; la vagina, con una pieza de pequeño tamaño en forma de flor de rosas y en color blanco; la abertura uretral, en forma de flor mosqueta, en color negro; y el clítoris, con un un objeto de circunferencia lisa blanca de pequeño tamaño. Además de estas obras de retrato sobre las tipologías de MGF, la exposición también incluía otras dos obras en las que la misma propuesta anónima de los retratos eran intervenidas con otros objetos que también tienen una fuerte carga expresiva en torno al tema de la MGF. Estos fueron objetos de cuchillas con manchas rojas y alfileres rojos, esparcidos por toda la zona del rostro, y objetos de alfiler con la bola superior en color rojo.

Para realizar la investigación, la artista había realizado entrevistas con mujeres que habían sufrido MGF -información disponible en el texto de presentación de la exposición-. Estas eran mujeres residentes en Reino Unido y de descendencia africana. La exposición también incluyó en sala los testimonios de estas mujeres, en forma de textos escritos con carácter poético. Así, al lado de cada retrato, también había

color. En este sentido, realiza una importante actividad de promoción de la investigación social y cultural a través de las artes. Página web oficial de la galería de arte Autograph ABP: https://autograph.org.uk/

R. Inter. Interdisc. Art\&Sensorium, Curitiba, v.7, n.1, p. 099 - 118 Jan.- Jun. 2020 
dispuesto un texto en lengua inglesa sobre fondo blanco, de pequeño formato. Estos textos estaban compuestos por fragmentos de entrevista a las mujeres. Fueron elaborados por la alista, según selección de los fragmentos más críticos y representativos de estos testimonios. Los textos estaban compuestos por segmentos cortos de apenas una línea que, en conjunto, narraban el origen de estas mujeres, el suceso de la MGF que cada una había vivida, y de las graves secuelas emocionales, psíquicas, biológicas, y también de dificultades para relaciones sociales, de pareja, sexuales, etc.

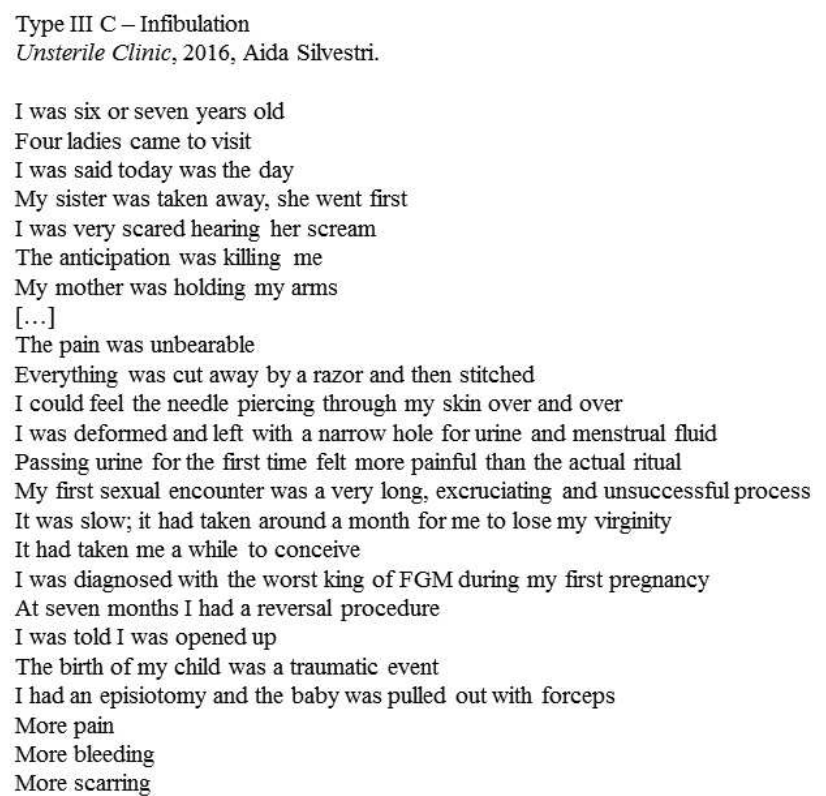

Imagen 2. Ejemplo de un texto de la exposición con el testimonio de mujer que ha sufrido MGF. 2016.

Aida Silvestri. Exposición Unsterile Clinic, Autograph ABP-Rivington Place, Londres. Imagen propia, 2016. Texto original en inglés, traducido por la autora al español.

La exposición ocupaba una única sala diáfana del edificio. Era de forma rectangular, de modo que la obra de Aida Silvestri se disponía a lo largo de los muros. El tratamiento estético de la sala era de carácter neutro: muros blancos lisos y suelo gris. La exposición no tenía un sentido único en alguna dirección concreta y, verdaderamente, podía verse de manera multidireccional. Sin embargo, para una mejor legibilidad de esta narración, se seguirá el sentido tradicional de lectura occidental, de izquierda a derecha. Al principio del primer muro, a la izquierda, se disponía un texto de presentación de la exposición. El texto aludía a varias cuestiones: comisariado, marco legal de la MGF en el Reino Unido, marco antropológico de la mutilación, ámbito territorial, motivaciones e intenciones de la artista, breve descripción de la obra expuesta, distinción de la aportación original de la exposición con respecto a otras anteriores de esta obra y propuesta a participar en una creación colectiva dentro de la muestra. A continuación, siguiendo el muro, empezaba la serie de obras de Aida Silvestri, y que se extendía a lo largo de los muros de la exposición. Para completar esta descripción, cabe mencionar que en el centro de la sala se disponía una mesa de vitrina. En esta se exponía otra serie de obras de vulva de la artista, pero sin retratos, representando otras diferentes tipologías de MGF que se realizan. 


\section{Exposición Itinerarios del vértigo en el Museo de Arte de Pereira (Colombia).}

Itinerarios del vértigo ${ }^{8}$ fue una exposición de arte con obra monográfica de la artista Sandra Silva y que pudo fue acogida en el Museo de Arte de Pereira9 (Pereira, Colombia), del 26 de junio al 16 de agosto de 2015. El conjunto de las obras y artefactos expuestos procedían de un mismo proyecto artístico, que la artista había realizado originalmente a lo largo del año 2014 en el marco de su investigación doctoral en Diseño y Creación de la Universidad de Caldas.

El tema general del proyecto fue los lugares del miedo y el colectivo travesti en la ciudad de Pereira, que la artista abordó según una investigación social basada en las artes (SILVA-CAÑAVERAL, 2019). Así, por un lado, y de acuerdo al sentido social de la investigación, la artista se basó en la propia población del lugar como informantes. Y, por otro lado, y de acuerdo al sentido artístico de la metodología de la investigación, la artista también elaboró unos artefactos de tipo visual y sonoro para recoger opiniones y testimonios de la población local. Estos artefactos también estuvieron expuestos en sala.

Los artefactos fueron de dos tipos: el Fobófono (titulado así por la arista) y el Mapa simbólico del miedo. El Fobófono consistió en un dispositivo de grabación de voz, que se accionaba pulsando un botón, y con el que los/as ciudadanos/as de Pereira iban grabando sus voces, libremente y de forma autónoma. Junto al Fobófono, la artista había indicado alguna señalética con preguntas, del tipo: “¿Qué es lo que le produce miedo en el sector del Parque de la Libertad?", de manera que la gente que quisiera participar, podía graban sus propias respuestas de forma abierta. El Mapa simbólico del miedo, por su parte, consistió en una imagen visual a color e impresa sobre vinilo, en la que estaba representado el callejero de la ciudad de Pereira. En esta ocasión, la artista invitaba a la gente a que indicaran sobre el Mapa aquellos lugares que relacionaba con el miedo. Para ello, junto al Mapa, la artista también había dispuesto una serie de pegatinas, y que tenían una representación icónica de diferentes miedos (robo de carros, atracos, indigencia, cosquilleo, venta y tráfico de drogas, entre otros).

\footnotetext{
${ }^{8}$ MUSEO DE ARTE DE PEREIRA. (18/07/2015). Sandra Silva: Itinerarios del vértigo [archivo de vídeo]. Disponible en: https://www.youtube.com/watch?v=evrv32F-_Xk [Consultado: 9/04/2020].

${ }^{9}$ El Museo de Arte de Pereira constituye el museo local y municipal de la ciudad de Pereira, con especial dedicación a la exposición de obras de carácter estético y visual. Está dirigido a artistas y/o temáticas regionales. En esta ocasión, este se presentaba como el lugar de mayor afinidad para exponer el trabajo artístico de Itinerarios del vértigo, según la artista había realizado una investigación local en la ciudad (el miedo en Pereira). Página web oficial del Museo de Arte de Pereira: https://museoartepereira.org/
}

R. Inter. Interdisc. Art\&Sensorium, Curitiba, v.7, n.1, p. 099 - 118 Jan.- Jun. 2020 

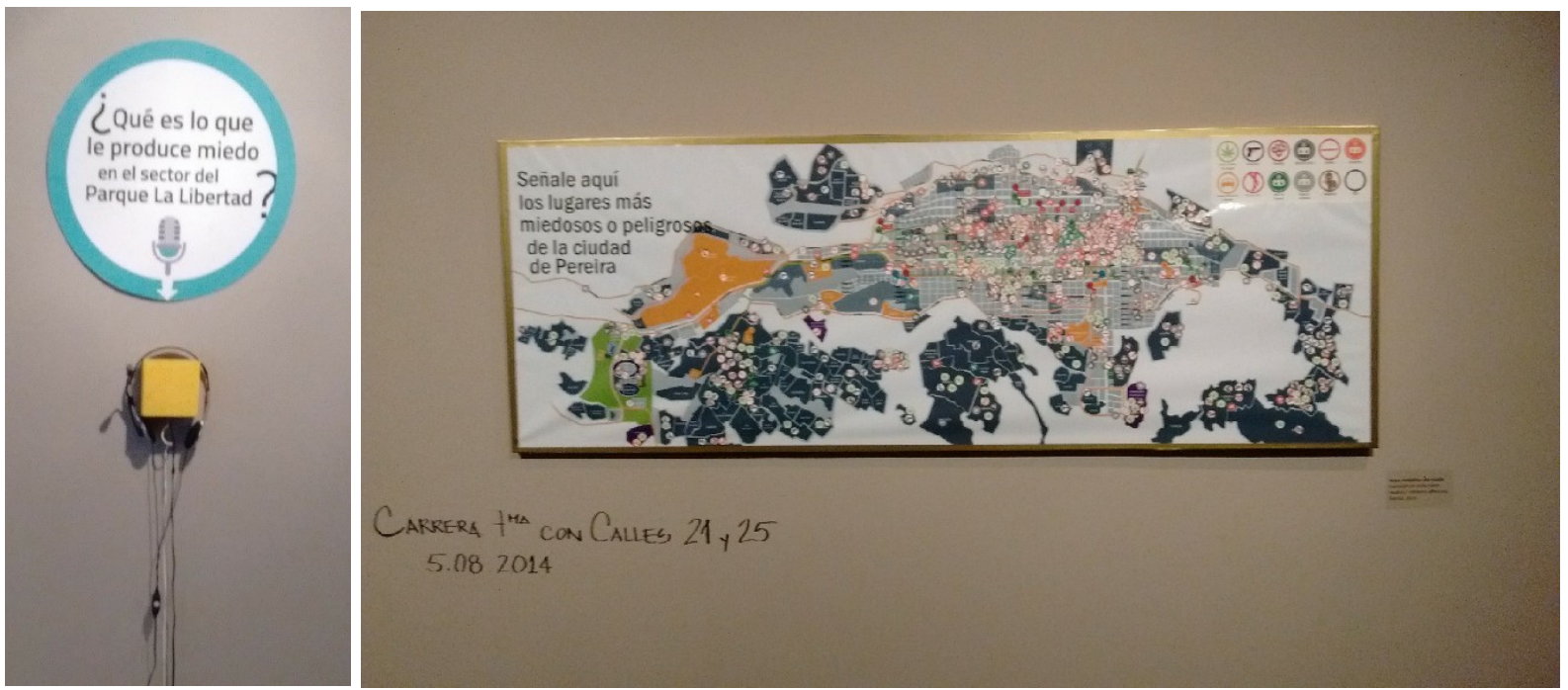

Imagen 3. Fobófono y Mapa simbólica del miedo. 2014. Sandra Silva. Exposición Itinerarios del vértigo, Museo de Arte de Pereira, Pereira. Imagen propia, 2015.

Respecto de las obras de arte de la exposición, se encontraton un total de tres obras: Crónica audiovisual "Días de Afrodita", un vídeo de tipo documental realizado por la artista sobre escenas cotidianas de la vida de personas travesti en Pereira y de cómo viven el ejercicio de la prostitución; Vestidos Orales, una instalación escultórica de maniquíes, ataviados según identidades, corporeidades y representaciones visuales del colectivo social travesti de la investigación; y el Libro de pensamiento y producción estéticoartístico, un libro de artista, que constituye la memoria de la investigación artística realizada, y donde se presentan las descripciones en texto escrito con las composiciones visuales de dibujos. 


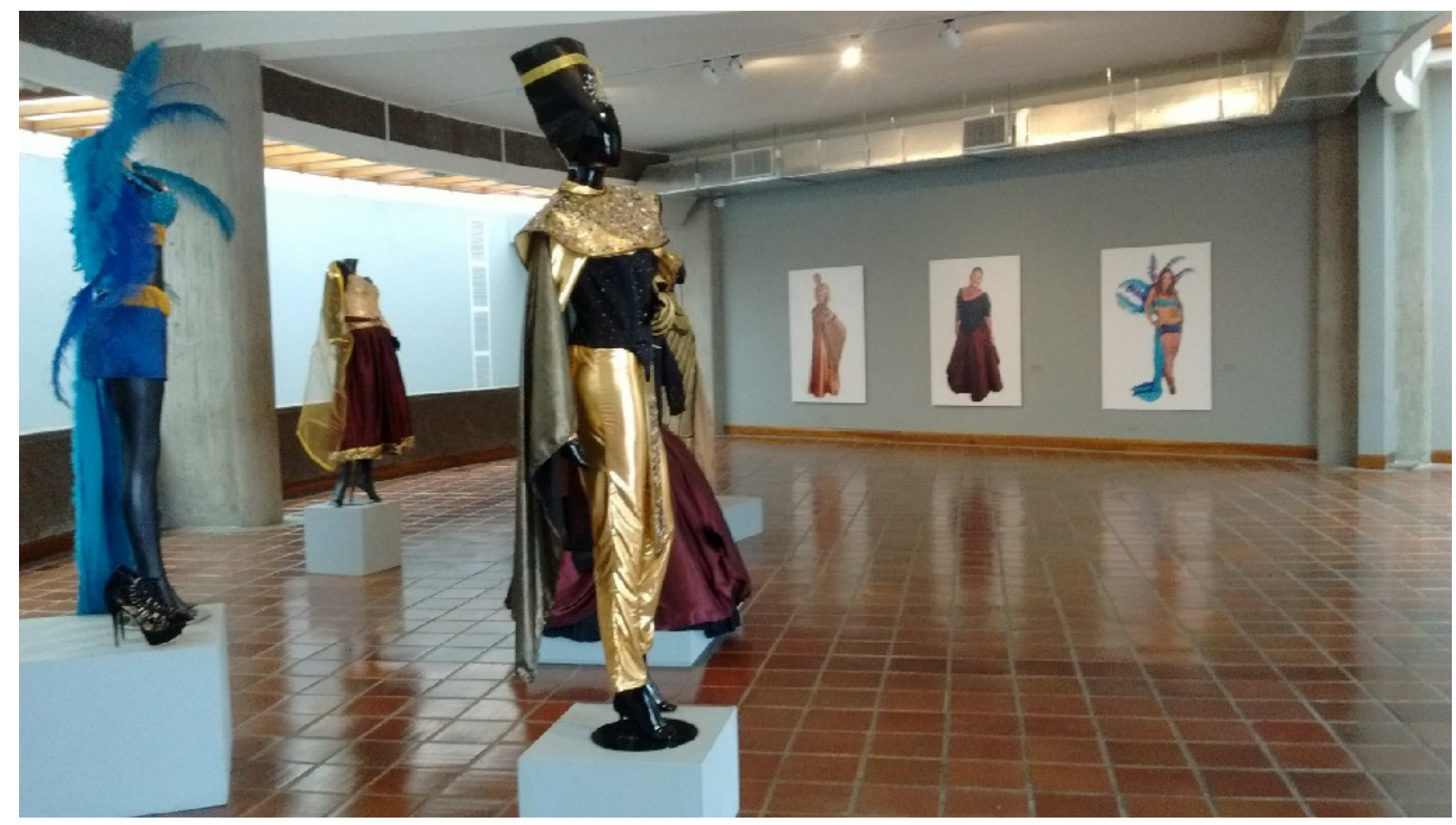

Imagen 4. Vestidos Orales (2014), de Sandra Silva. Exposición "Itinerarios del vértigo", Museo de Arte de Pereira, Pereira. Fuente: imagen propia, 2015.

En esta exposición destaca también un mapa visual de grandes dimensiones, dibujado sobre uno de los muros, a la entrada de la exposición. Está elaborado a base de formas geométricas y líneas unidas entre sí, que a su vez ordenan y relaciona una serie de palabras clave. En este mapa, podía leerse: "INVESTIGACIÓN BASADA EN LA CREACIÓN”; arriba, "ITINERARIOS DEL VÉRTIGO; debajo, "A partir de LO RELACIONAL y LO CONTEXTUAL"; en los laterales, "ACONTECIMIENTOS ESTÉTICO-ARTÍSTICOS”, junto a "1.INSTALACIÓN VESTIDOS ORALES”, “2.CRÓNICA AUDIOVISUAL DÍAS DE AFRODITA" y "3.LIBRO DE PENSAMIENTO Y PRODUCCIÓN ESTÉTICO-ARTÍSTICO"; y, también en los laterales, "PROVOCACIONES ESTÉTICO ARTÍSTICAS, junto a "1.MAPA SIMBÓLICO DEL MIEDO”, “2.FOBÓFONO” y “3.REGISTRO AUDIOVISUAL VESTIDOS ORALES”. 


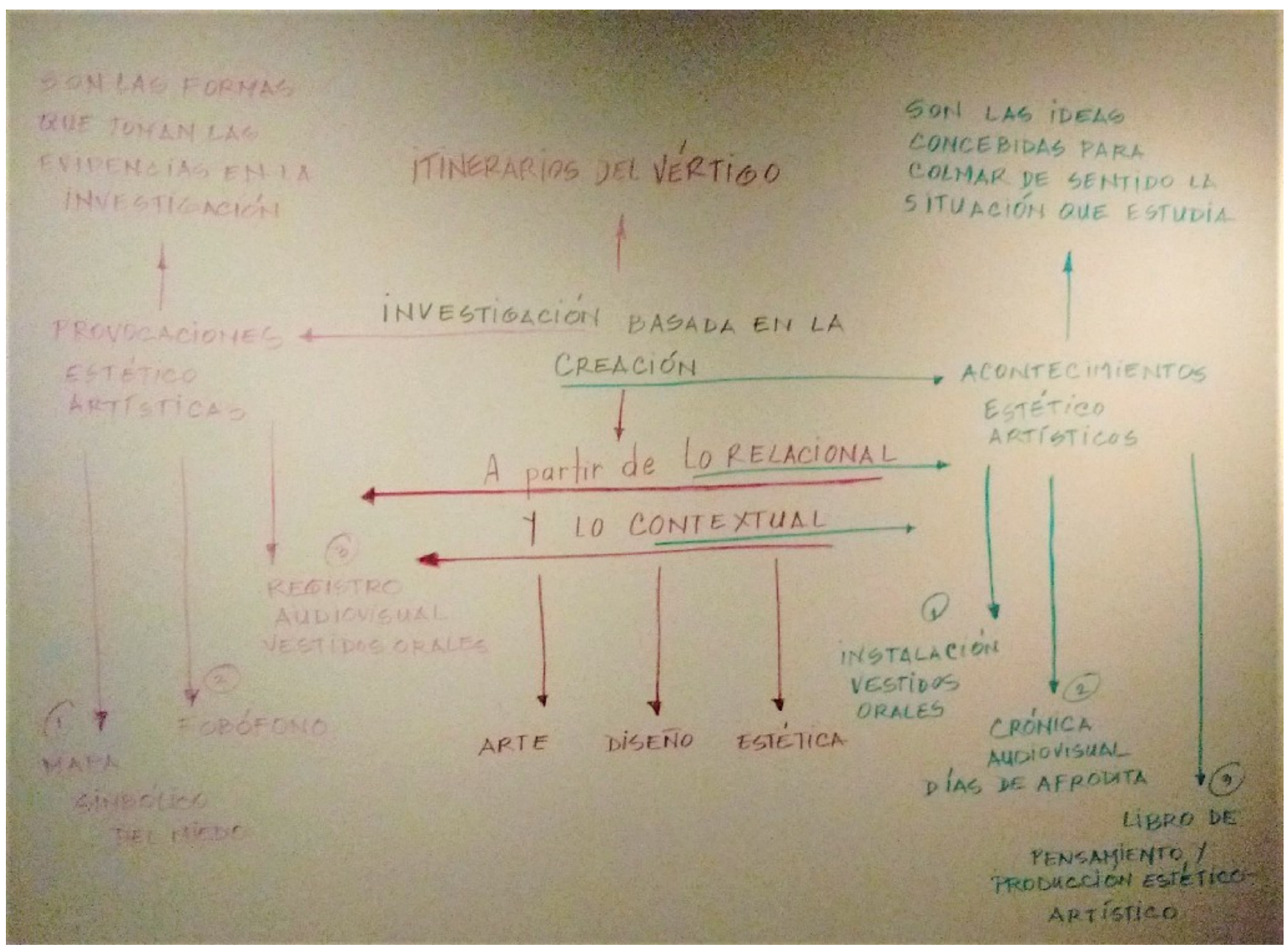

Imagen 5. Mapa conceptual (2015), de Sandra Silva. Exposición "Itinerarios del vértigo", Museo de Arte de Pereira, Pereira. Fuente: imagen propia, 2015.

La exposición ocupó un total de dos pisos o niveles arquitectónicos del Museo, entre un total de tres salas que se recorrían de forma continua unas de otras. La primera sala estaba dedicada a la presentación y contextualización de las obras; así, lo primero que se encontraba era el un mapa conceptual sobre la investigación artística de Sandra Silva, un texto de presentación escrito por el director del programa de doctorado y una mesa sobre la que poder consultar tanto la memoria de doctorado, como un dispositivo de ordenador donde realizar otras consultas dedicado a este proyecto. El texto de presentación estaba escrito por el Director Doctorado en Diseño y Creación de la Universidad de Caldas (D. Adolfo León Grisales Vargas), en cuyo marco de estudios de postgrado la artista realizó la obra. En este se refiere a varios aspectos destacados sobre el tema del miedo de la ciudad que investiga la artista. A continuación, en uno de los laterales se habría un pequeño corredor, en cuyos muros se disponían el Mapa simbólico del miedo y el Fobófono, así como también daba acceso a otra sala donde se encontraba la proyección audiovisual de "Días de Afrodita". Para terminar el recorrido de la exposición, al final de este pasillo, unas escaleras daban acceso al segundo piso; a lo largo de toda la planta se extendía una amplia sala diáfana en la que se encontraban distribuidas por el espacio los diferentes maniquíes de la obra Vestidos Orales. La estética arquitectónica de las salas de esta exposición sigue la estética que es común a todo el 
edificio del Museo, y que responde a un tratamiento neutral, según muros lisos en color gris -incluso en homigón visto-, y solería de baldosas marrones.

\section{Procedimiento}

Para la recogida de datos y debido al carácter físico y visual de la muestra, se realizó una observación directa (ANGROSINO, 2012; DÍAZ, 2012) en los sitios de las exposiciones, respectivamente, y empleando técnicas de grabación fotográfica y videográfica ${ }^{10}$. Para la recogida de datos en la exposición Unsterile clinic (2016) en el Rivington Place de Londres, la investigadora realizó una estancia larga internacional de 3 meses en el Educational Studies Departament of Golsmiths-University of London ${ }^{11}$, del 1 de septiembre al 30 de noviembre de 2016, periodo durante el cual realizó, a su vez, varias y sucesivas visitas al sitio de la exposición. De igual modo y para la recogida de datos en la exposición Itinerarios del vértigo (2015) en el Museo de Arte de Pereira, la investigadora realizó una estancia larga internacional de 3 meses en el Departamento de Artes Visuales y Estética de la Universidad del Valle ${ }^{12}$ (Cali, Colombia), periodo durante el cual realizó, a su vez, varias y sucesivas visitas al sitio de la exposición.

La interpretación de las exposiciones de arte está basada en las cuestiones relativas a la investigación artística. Esta concierne a cuestiones ontológicas, epistemológicas y metodológicas de la investigación (BORGDORFF, 2006), pero no en un sentido sistematizado del método, sino de la elaboración de los sentidos (GUATTARI, 1993; CONDERANA, 2016). De este modo, una de las cuestiones principales de la investigación artística es el reconocimiento del contexto en que sucede la investigación. Este no solo se identifica con un sitio o lugar concreto, sino que, desde un enfoque holístico, se refiere al conjunto del imaginario cultural al que se circunscriben los/as artistas, situaciones, temas de estudio y proceso del trabajo (CALDERÓN Y HERNÁNDEZ, 2019). A este respecto, Toledo habla de una relación entre arte y vida, entendiendo que la investigación artística ya no pertenece exclusivamente a la cuestión estética, sino "al estudio del mundo, donde se entretejen relaciones de sentido entre arte, vida y política" (TOLEDO, 2012, p. 47). Desde esta idea, la investigación artística deja de ser solamente una cuestión objetual, esto es, de técnicas y procedimientos. Se adoptan como temas de estudio aquellos del arte, pero también los temas sociales, culturales, políticos y, en suma, a la investigación artística le interesa sobre todo la condición humana. Así, son tema de estudio de la investigación artística situaciones, fenómenos, eventos, etc. Por tanto, y de acuerdo a lo anterior, las cuestiones de la investigación artística residen en la epistemología de la investigación. Como señala Zúñiga: "los procesos de percepción, cómo es que se ve el mundo, cómo lo conozco y me lo apropio" (ZÚÑIGA, 2015, p. 70).

Se distinguen diferentes enfoques de la investigación artística, y que también condicionan las metodologías y materiales de la investigación. En este sentido, existe un intenso debate de terminología, según investigación para las artes, investigación en las artes, investigación basada en las artes, etc. (BORGDORFF, 2006; HANNULA, SUORANTA y VADÉN, 2014). El presente trabajo no tiene el objetivo de profundizar en este debate. Sin embargo, es pertinente anotar que los diferentes enfoques condicionan a su vez las metodologías y materiales de la investigación artística. Por poner dos ejemplos

\footnotetext{
${ }^{10}$ La grabación fotográfica y videográfica estaba permitida libremente en ambos sitios de la muestra.

${ }^{11}$ Página web oficial del Educational Studies Departament of Golsmiths-University of London:

https://www.gold.ac.uk/educational-studies/

${ }_{12}$ Página web oficial del Departamento de Artes Visuales y Estética de la Universidad del Valle: http://artesvisualesyestetica.univalle.edu.co/
}

R. Inter. Interdisc. Art\&Sensorium, Curitiba, v.7, n.1, p. 099 - 118 Jan.- Jun. 2020 
más directamente relaciones con los casos de este estudio. Por un lado, en la investigación en las artes no se distingue el sujeto de estudio del objeto de estudio, esto es, entre el/a investigador/a y la práctica artística, de modo que la información esencial de la investigación residen en la propia experiencia de vida que experimenta el/a investigador en el transcurso de la investigación, además de la documentación (en un sentido amplio) y los tratamientos materiales y técnicos que se derivaban. Por otro lado, en la investigación basada en las artes, el tema de estudio no es propiamente artístico, de manera que se emplea la práctica artística para articular los sentidos y significados que se van encontrando en la investigación (como, por ejemplo, la investigación social basada en las artes, y alguno de cuyos derivados lo constituyen movimientos como el artivismo y el arte para la justifica social; o la investigación educativa basada en las artes); de este modo, la información social recogida en el trabajo de campo constituye una parte esencial en la investigación (entrevistas, observación directa, residencia en el lugar, etc.).

\section{Resultados}

En este apartado se presentan los resultados de la interpretación de las dos exposiciones de arte de la muestra, respecto de qué contenidos sobre investigación artística están presentes en la exposición, y sobre cómo se ha resuelto la musealización de estos para introducirlos en las sala de las exposiciones.

Los resultados para la interpretación de las dos exposiciones de la muestra sobre contenidos de investigación artística son:

- $\quad$ Contenidos de contextualización y justificación de la investigación.

- Contenidos de testimonios de los informantes de la investigación.

- Contenidos de la metodología de la investigación.

- Contenidos de los resultados de la investigación.

A continuación se presentan en detalles los resultados para cada uno de los contenidos anteriores.

Los contenidos de las exposiciones que corresponden con contextualización y justificación de la investigación se han encontrado en forma de texto de presentación de la exposición. Esta forma se repite en ambos casos de la muestra. En la exposición Unsterile clinic, el texto comienza con un párrafo de la comisaria, Renée Mussai ${ }^{13}$, donde pone en relación los derechos humanos con la fotografía, para una denuncia urgente de esta situación, y que afecta globalmente a mujeres y niñas. Informa acerca del marco legal de la MGF en el Reino Unido ${ }^{14}$, señalando que se convirtió en delito en 1985, que en 2003 esta ley fue extendida a todas las personas de nacionalidad británica y con residencia permanente en el país, y que en 2015 fue reforzada para ser objeto de esta ley también fuera del país. Sitúa un marco antropológico sobre la MGF, respecto de cuestiones de identidad, tradición, religión y de las fatales consecuencias en los cuerpos y vidas de niñas y mujeres (fuertes dolores que causan mareos, infecciones vaginales graves, problemas urinarios, de infertilidad, de embarazo y alumbramiento, de identidad y de relación sexual), (ÁLVAREZ, 2001). Sitúa el ámbito territorial de esta práctica, que históricamente se originó en zonas de África y Medio-Este, pero que, actualmente, los movimientos migrantarios lo han extendido por todo el planeta y se estima que hoy en día afecta a alrededor de 125 millones de personas. El texto de presentación de la exposición también se refiere a las motivaciones de la artista para este trabajo. Cuenta

\footnotetext{
${ }^{13}$ Reseña breve sobre Renée Musssai en la página web oficial de Autograph ABP: https://autograph.org.uk/about-us/contact

${ }^{14}$ Declaración oficial en contra de la mutilación genital femenina del Ministerio de la Salud del Gobierno del Reino Unido: https://www.gov.uk/government/publications/statement-opposing-female-genital-mutilation
}

R. Inter. Interdisc. Art\&Sensorium, Curitiba, v.7, n.1, p. 099 - 118 Jan.- Jun. 2020 
que se debe a la experiencia personal de Aida Silvestri y a la necesidad de dar visibilidad a este colectivo (solo en el Reino Unido, los casos de MGF tan solo son diagnosticados durante el embarazo o el parto). Las intenciones son las de concienciar a las mujeres de no hacerlo, así como empoderar al personal médico para que hable y aborde el tema abiertamente, y contribuir así con estas herramientas visuales también a la diversidad tipológica de los casos de MGF, adaptada a su vez -según informa este texto- al listado de la Organización Mundial de la Salud. Respecto del proyecto artístico, se informa que se trata de un encargo original de la entidad Autograph ABP a la artista en 2016. En último lugar, se invita al público a participar de una propuesta artística colectiva en la exposición, justificada con motivo del $2^{\circ}$ aniversario de "Girl Summit", organizado por el gobierno del Reino Unido en colaboración con UNICEF.

En la exposición Itinerarios del vértigo, por su parte, el texto se refiere a varios aspectos destacados sobre el tema del miedo de la ciudad que investiga la artista: el miedo atraviesa toda la ciudad y no se refiere a rincones concretos; el miedo ha conducido a la población a recluirse en espacios privados de interior, de modo que el espacio exterior de la ciudad ha quedado deshabitada de señales de identidad; la desconfianza que existe entre la población respecto del colectivo travesti y que el Director (autor del texto y que se identificaba en líneas anteriores) relaciona con cierto recelo o envidia porque se muestran tal y como son.

Los contenidos de las exposiciones que corresponden con los testimonios de los/as informantes de la investigación adquieren formas muy diversas en cada una de las exposiciones. En Unsterile clinic adquieren forma de textos poéticos, mientras que en Itinerarios del vértigo se tratan de los artefactos del Fobófono y del Mapa visual del miedo. Ambos casos tienen en común que todas estas formas están elaboradas por las artistas de la exposición. En este sentido, resulta importante destacar que no se tratan de dispositivos de información elaborados por el comisariado o la institución. En Unsterile clinic, los textos provinenen de la transcripción de entrevista a informantes de la investigación de la artista. Sin embargo, están compuestos por una selección de fragmentos breves, según los más críticos, representativos y expresivos del testimonio de historia de vida de las mujeres infomantes. Así, estos textos se estructuran en fragmentos libres que ocupan apenas una línea de extensión. Así, estos textos poéticos en la exposición Unsterile clinic introducen los datos recogidos de las informantes de la investigación realizada por la artista Aida Silvestri.

Por su parte, en Itinerarios del vértigo, la artista elaboró dos artefactos interactivos para el registro de opiniones y experiencias de la población de Pereira respecto de cómo viven el miedo en la ciudad. Es en este sentido que estos dispositivos constituyen los testimonios de los informantes de la investigación. Para el "Mapa visual del miedo", las personas debían colocar una pegatina del tipo de miedo en la zona de la ciudad donde conocen que este tiene lugar; para el Fobófono, debían pulsar un botón para grabar su voz oral y dejar su opinión y experiencias sobre el miedo en la ciudad. Este artefacto de grabación y de escucha permitan el registro original de las voces orales de los informantes de la investigación, así como su posterior escucha, conectando estos a unos auriculares o altavoces externos. De este modo, el resultado de ambos artefactos es que estos contienen el conjunto de testimonios de la población de Pereira en torno al miedo.

Los contenidos de las exposiciones que corresponden con la metodología de la investigación solo se han identificado para uno de las exposiciones: en Itinerarios del vértigo. Estos contenidos se han encontrado articulados en la exposición en forma de mapa visual, y que estaba representado sobre uno de los museos. Los mapas visuales se utilizan para la ordenación semántica de una información amplia y compleja. Están constituidos a base de palabras clave o unidades simbólicas, y basados en principios del lenguaje visual (CAMPOS, 2005), según los círculos concentran la atención y las líneas conectan; la aproximación de

R. Inter. Interdisc. Art\&Sensorium, Curitiba, v.7, n.1, p. 099 - 118 Jan.- Jun. 2020 
elementos, afinidad. El resultado de estos mapas es articular una estructura o red semántica de elementos y conceptos relacionados entre sí (OJEDA, et al., 2007). De esta forma, la zona central y principal del mapa representa el tipo de metodología de investigación que adoptó la artista para su proyecto: "INVESTIGACIÓN BASADA EN LA CREACIÓN", y que corresponde con la metodología también conocida como investigación basada en las artes (MCNIFF, 1998). En este sentido, también se alude a algunos de los principios teóricos o características procedimentales del método de investigación-acción (ANDER-EGG, 1990; COLMENARES, 2012): "A partir de LO RELACIONAL y LO CONTEXTUAL", de acuerdo a una de las teorías artísticas actuales de mayor repercusión internacional, que ha sido la "Estética Relancional", pronunciada por Nicolas Bourriaud (2001).

En los laterales del mapa se extiende una información más específica sobre el proceso artístico realizado, y que aluden, por un lado, a las obras de arte que se elaboraron en el marco del proceso y, por otro, a los artefactos que articuló la artista para recoger testimonios de los informatnes: "ACONTECIMIENTOS ESTÉTICO-ARTÍSTICOS" ("1.INSTALACIÓN VESTIDOS ORALES", "2.CRÓNICA AUDIOVISUAL DÍAS DE AFRODITA" y "3.LIBRO DE PENSAMIENTO Y PRODUCCIÓN ESTÉTICO-ARTÍSTICO”) y "PROVOCACIONES ESTÉTICO ARTÍSTICAS" ("1.MAPA SIMBÓLICO DEL MIEDO”, “2.FOBÓFONO” y “3.REGISTRO AUDIOVISUAL VESTIDOS ORALES"). Inmersa en un marco teórico relacional y contextual de la metodología de la investigación, como se indicaba anteriormente, las obras se realizan en procesos colectivos y de colaboración entre la artista y el grupo social de la investigación -colectivo travesti en Pereira, en este caso- (ZÚÑIGA, 2015; GUATTARI, 1993). A este respecto es que la artista utiliza, y con pertinencia, el término "acontecimientos estético-artísticos", para así aludir al carácter procesual y grupal del proceso. Por otro lado, el término de "provocacaciones estético artísticas" destaca el compromiso social que es implícito de la investigación basada en las artes (TOLEDO, 2012).

\section{Consideraciones finales}

Permitan los/as lectores empezar la presentación de esta discusión de los resultados y conclusiones de este trabajo utilizando la metáfora de una hoja (ver Imagen 6). La Imagen 6 no es un hoja. Es la representación de una hoja -como pronunció originalmente René Magritte sobre la representación de una pipa-. Esa parte de la hoja que queda en la sombra, boca abajo y, en este sentido, acaba siendo el lugar secundario de la hoja. Sin embargo, es la cara de la hoja donde mejor pueden verse sus nervios por los que circula la savia. Me pregunto, entonces, si precisamente por este motivo, ¿no debería ser esta la cara principal de hoja? En un sentido metafórico, las caras más visibles del ámbito de las artes son los escenarios, las pantallas de cine y de televisión, y las exposiciones de arte, entre otras muchas. Como sucede en la hoja, la verdad queda oculta bajo la sombra. En un escenario sería todo aquello que sucede detrás del telón: el guion, la producción, los ensayos, los remiendos al atrezo, etc.; en una película sería la parte oculta tras la pantalla: el making-of, las tomas falsas, etc.; y en una exposición de arte, ¿qué sería? 


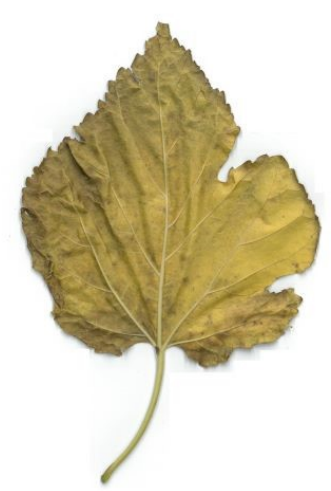

Imagen 6. Representación fotográfica del envés de una hoja. Imagen propia, 2020.

Con esta metáfora entre el envés de una hoja y la exposición de arte quisiera poner el foco sobre todo aquello que tradicionalmente quedaba en la sombra de la exposición de arte, que no está presente y no se incluye en las salas de exposición, pero que han configurado las obras y elementos que se exponen: la historia de vida de los/as autores/as (y no solo el currículum de méritos que la justifican); las referencias artísticas, teóricas, históricas y culturales que sus autores/as han considerado en el proceso de creación; las cuestiones técnicas y materiales del trabajo artístico realizado; las consideraciones vivenciales, subjetivas y teóricas que fueron dando lugar a la obra, y las decisiones que sus autores/as fueron tomando a lo largo del proceso. En definitiva, todo aquello que en un proyecto de investigación constituye el marco teórico, la metodología y los resultados.

En el marco de la investigación, Aida Silvestri accedió a entrevitar a mujeres que habían sufrido MGF. En cada caso, relataron cuándo les ocurrió, cuáles fueron las circunstancias, cómo se sintieron y quiénes estuvieron con ella y cómo les afectó posteriormente a varios niveles, de salud, maternidad, de relaciones sociales de pareja, de relaciones sexuales, etc. El acceso a esta información es privado y personal, que la artista consiguió realizando una importante labor de confianza y reconocimiento por parte de estas mujeres. Así, la obra tampoco se expone sola o independiente a estos relatos, si no que junto a cada una se incluyen fragmentos de estos testimonios desgarradores. En la exposición, el relato de estas mujeres cobra tanta imporancia como la obra misma. Si bien las obras suponen los objetos de carácter estético de la exposición, los relatos las convierten en un conocimiento contrastado y crítico, por el que el caso toma un cariz social. El formato de incluir los relatos podría haber sido otro diferente al texto escrito (podían haber sido también audiciones de voz, entre otras posibilidades). La importancia que destaca es que, ambos elementos, están presentes en la sala y toman su espacio y atención visual y expositiva, formando parte de la configuración misma de la exposición en su conjunto. Si en la exposición se obviaran los relatos, la muestra plástica perdería la casuística situacional y "real" que la originaron, en el sentido de los grupos sociales actuales a los que afecta y que la convierten, también, en una herramienta de denuncia social.

Así, entendemos que musealizar la investigación artística en la exposición supone introducir en sala contenidos que informan sobre el proceso realizado. En un sentido más categórico de la investigación, se podría pensar en contenidos que informen sobre las diferentes fases de la investigación. Sin embargo, 
y como se apuntaba anteriormente respecto de la investigación artística, aquí los métodos no se abordan de manera sistematica, sino que la investigación artística atiende a cuestiones epistemológicas de un conocimiento holístico. Por tanto, lo que interesa para la musealización de la investigación artística, es introducir y hacer visible el compendio de relaciones, sentidos e informaciones que han trabajado los/as artistas en el proceso y que, por extensión, han nutrido de contenido y significado los resultados de las obras que se exponen.

Los dos casos de exposición de la muestra se refieren a una investigación social basada en las artes (LEAVY, 2018; MCNIFF, 1998). Este tipo de investigación adopta técnicas etnográficas de documentación y recogida de datos, como entrevista, observación directa, acción-participación, etc. Así, en ambos casos y desde una musealización de la investigación artística, se introducen en sala contenidos de testimonios de los informatnes de la investigación, basados en dispositivos artísticos también: los textos poéticos con los testimonios de mujeres víctimas de MGF en el caso de la exposición Unsterile clinic, y "Mapa simbólico del miedo" y "Fobófono" con los testimonios y opiniones de ciudadanos/as en Perira en el caso de la exposición Itinerarios del vértigo. También se introducen contenidos de la metodología de la investigación, aunque esta vez tan solo en el caso de Itinerarios del vértigo, y que se articula en forma de mapa visual, lo que exige a su vez un conocimiento previo especializado para tu interpretación.

Sin embargo, ninguno de los casos de exposición de la muestra incluyen contenidos explícitos relativos a las lógicas de creación de las obras. En la exposición Unsterile Clinic (2016) de Aida Silvestri en Autrograph ABP-Rivington Place, Londres, (Reino Unido), las cualidades plásticas y visuales del arte combinan la lógica racional de la representación de la morfología de vulvas de mujeres de MGF, con la lógica sensible del tratamiento semiótico y simbólico de los elementos y sus materiales. Respecto de la obra, para la representación de los órganos sexuales femeninos se utiliza material de cuero en color carne; para la vagina, la flor de una rosa en color blanco y que brilla; para la abertura uretral, una flor de mosqueta en negro; y el clítoris, una circunferencia blanca. Este conjunto de elementos y sus lenguajes visuales y artísticos, ponen de manifiesto la condición femenina y de mujer de color en las dramáticas vivencias la MGF; la corporeidad humana del problema, la contemporaneidad de la situación y la delicadez y atención sensible y de empatía con las personas afectadas en primera persona con que debe abordarse este tema. En Itinerarios del vértigo, la información disponible acerca del cómo y del porqué de la obra Vestidos Orales no se encuentra inserta en sala, sino en una página web personal de la artista y relativa al proyecto realizado.

Musealizar la investigación artística en la exposición de arte supone desplazar la exclusividad del valor estético de las obras hacia otros valores culturales (FREITAS, BARRETO, 2019). En este sentido, supone destacar también otros valores de la producción artística como valores epistemológicos, documentales, históricos y sociales, entre otros. Los contenidos de las investigaciones artísticas que se han musealizado en las salas de las dos exposiciones de la muestra han sido principalmente de carácter social, según la justificación social y política de la investigación y los testimonios de los informantes. De este modo, la investigación basada en las artes resulta favorable a una musealización en la exposición de arte. Sin embargo, los contenidos relativos a las lógicas del proceso de creación de las obras, resultan invisibles para las salas de exposición. Comúnmente, toda esa información constituye los catálogos de exposición y, más recientemente, las audio-guías, aplicaciones informáticas, material digital en línea, etc. Los precios de catálogos de arte rondan entre los 20 y 50 euros para exposiciones locales; para exposiciones de museos o centros nacionales, los catálogos pueden alcanzar precios de 100 y 200 euros. En los contenidos de estos catálogos se encuentra una extensa argumentación del fenómeno cultural de las obras, 
también sobre sus autores, procesos de creación y contextos, que incluyen un buen repertorio de referenicas y alusiones a los marcos teórico-culturales y a los contextos histórico-artísticos, tanto del momento de realización por los/as artistas sus obras, como de las miradas actuales en torno a ellos por los/as historiadores/as y teóricos/as del arte. También en los contenidos de audio-guías, Apps informáticas y otro material en línea, aunque en estos casos, los contenidos suelen ser más parciales y escuetos.

Las posturas respecto de incluir o no información interna son divergentes, entre quienes consideran que debe ser mínima, según motivos de cansancio en los/as visitantes, de perturbación la atención visual de la obra y de imposición de una interpretación sobre la obra; y quienes consideran que la información interna constituye uno de los sentidos de la exposición de arte -especialmente en el caso de los museos(ÁVILA, 2003, p. 354). Los dispositivos estético-artísticos no funcionan como información interna al uso, según cartelería o señalética (TIRADO, 2019). No responden a un tratamiento didáctico de la información, dado que no tienen carácter explicativo. Como tampoco se tratan de bocetos de las obras, dado que no representan material preparatorio de los discursos artísticos de las obras. Por tanto, en este trabajo se entiende que la interacción estos dispositivos y el público es de tipo deductiva, en el sentido de que implica un razonamiento entre sus contenidos y los de las obras expuestas, de modo que las lógicas de sentido entre unas y otras no están resueltas.

La creación artística actual sucede en medio de procesos psicológicos y cognitivos del conocimiento, y ya no concierne a una búsqueda de certezas canónigas de lo bello o de lo artístico, sino al desarrollo de un conocimiento de cada uno/a para crear sentidos sobre una realidad dada (ARAÑó, 2005), y a la capacidad creativa de la interacción y del diálogo socio-cultural (GUASH, 2016). De este modo, las prácticas de arte contemporáneo y de arte actual han desplazado definitivamente la producción artística por encargo del mecenas, hacia por una producción desde el "yo" del artista -desplazando también los temas, géneros y técnicas canónigas del arte, por otros de los contextos socio-culturales actuales con los que los/as artistas conviven y se debaten- (BOURRIAUD, 2001, 2003). Por tanto, si en el campo de la producción artística contemporánea y actual se han desplazado los temas y procesos, las formas de exponer el arte también debe dejar paso a estos otros valores en torno al arte y su producción, de modo que la exposición no se focalice exclusivamente en los valores estéticos de las obras. Así, las exposiciones dedicadas al arte actual enfrentan el reto de, junto a las obras, dar cuenta de las lógicas de su creación.

\section{Referencias bibliográficas}

ALONSO, L. Museología y Museografía. 2a ed. Barcelona: Ediciones del Serbal, 1999, 2001.

Álvarez Degregori, M.C. Sobre la mutilación genital femenina y otros demonios. Bellaterra, España: Universitat Autònoma de Barcelona, 2001.

ANDER-EGG, E. Repensando la Investigación Acción-Participación. Buenos Aires: Grupo Editorial Lumen Hvmanitas, 1990, 2003.

ANGROSINO, M. Etnografía y observación participante en investigación cualitativa. Madrid: Morata, 2012

ARAÑÓ, J. C. Estructura del conocimiento artístico. In: MARÍN VIADEL, R. (ed.). Investigación en educación artística: temas, métodos y técnicas de indagación sobre el aprendizaje y la enseñanza de las artes y culturas visuales. Granada, España: Universidad de Granada, 2005. 19-42.

R. Inter. Interdisc. Art\&Sensorium, Curitiba, v.7, n.1, p. 099 - 118 Jan.- Jun. 2020 
AUTOGRAPH ABP. (2016). Aida Silvestri: Unsterile Clinic. Disponível em <http://autographabp.co.uk/exhibitions/unsterile-clinic>. Acesso em 11/03/2020.

ÁVILA, A. El Arte y sus Museos. Barcelona: Editorial del Serbal, 2003.

BORGDORFF, H. El debate sobre la investigación en las artes. Cairon: revista de ciencias de la danza, 13: 25-46, 2006.

BOURRIAUD, N. Esthétique Relationnelle. Dijon, Francia: les presses du réel, 2001, 2006.

BOURRIAUD, N. Postproduction. La culture comme scénario: comment l'art reprogramme le monde contemporaine. Dijon, Francia: les presses du réel, 2003, 2009.

CALDERÓN GARCÍA, N.; HERNÁNDEZ HERNÁNDEZ, F. La investigación artística: un espacio de conocimiento disruptivo en las artes y en la universidad. Barcelona: Octaedro, 2019.

CAMPOS ARENAS, A. Mapas Conceptuales, Mapas Mentales y otras formas de Representación del Conocimiento. Bogotá: Editorial Magisterio, 2005.

COLMENARES, A.M. (2012). Investigación-acción participativa: una metodología integradora del conocimiento y la acción. Voces y Silencios: Revista Latinoamericana de Educación, vol. 3, núm. 1, 102115, 2012. Disponível em <https://revistas.uniandes.edu.co/doi/pdf/10.18175/vys3.1.2012.07>. Acesso em 15/03/2020.

CONDERANA, J. A. (coord.). Giros epistemológicos de las artes: la creación de significado. Madrid: Ediciones Asimétricas, 2016.

DÍAZ, A. El taller del etnógrafo: materiales y herramientas de investigación en Etnografía. Madrid: UNED-Universidad Nacional a Distancia, 2012.

DUBÉ, P. Exponer para ver, exponer para conocer, Museum International, vol. 185, núm. 1, 4-5, 1995.

FREITAS, A.C.; BARRETO, K.M. O Processo de Formação do Acervo e da Instituição:

Novomuseu/Mon. Art \& Sensorium, vol. 6, núm. 1, 1-13 2019. Disponível em

$<$ http://periodicos.unespar.edu.br/index.php/sensorium/article/view/2475/1811>. Acesso em $15 / 03 / 2020$.

GUASCH, A. M. El arte en la era de lo global: 1989-2015. Madrid: Alianza Editorial, 2016.

GUASCH, A. M. (1996). El arte de los ochenta y las exposiciones. Reflexiones en torno al fenómeno de la exposición como medio para establecer los significados culturales del arte, D'Art, 22, 1996, 143-159.

GUATTARI, F. El constructivismo guattariano: Un proyecto etico-estetico para una era post-media. Cali, Colombia: Ediciones Universidad del Valle, 1993.

HANNUlA, M.; SUORANTA, J.; VADÉN, T. Artistic Research Methodology: Narrative, Power and the Public (Critical Qualitative Research). Nueva York: Peter Lang, 2014.

HOOPER-GREENHILL, E. Museums and the shaping of knowledge. Londres: Routledge, 1992.

LEAVY, P. (ed). Handbook of Arts-Based Research. New York: The Guilford Press, 2018.

R. Inter. Interdisc. Art\&Sensorium, Curitiba, v.7, n.1, p. 099 - 118 Jan.- Jun. 2020 
LORENTE, J. P. (2015). Estrategias museográficas actuales relacionadas con la museología crítica,

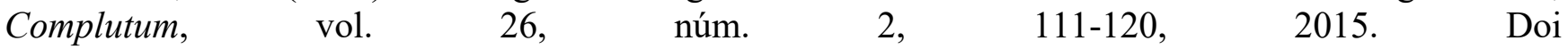
$<$ http://dx.doi.org/10.5209/rev_CMPL.2015.v26.n2.50422>.

LORENTE, J. P. La "nueva museología” ha muerto, ¡viva la "museología crítica”!. In: Almazán, D; Lorente, J.P. Museologia, crítica y arte contemporáneo. Zaragoza, España: Universidad de Zaragoza, 2013. 13-25.

LORENTE, J. P. Los museos de arte contemporáneo: Noción y desarrollo histórico. Gijón, España: Trea, 2008.

MARTÍN, A.M. La información, un recurso en alza: el futuro de la documentación en los museos,

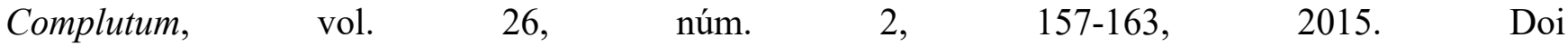
$<$ http://dx.doi.org/10.5209/rev_CMPL.2015.v26.n2.50426>.

MASSERONI, A. Metodología y métodos: diseños de investigación comparativa. Buenos Aires: Mnemosyne, 2010.

MCNIFF, S. Art-Based Research. Londres: Jessica Kingsley Publishers, 1998, 2006.

MUSEO DE ARTE DE PEREIRA. Sandra Silva: Itinerarios del vértigo. Disponível em $<$ https://www.youtube.com/watch?v=evrv32F__Xk>. Acesso em 8/03/2020.

OJEDA CABRERA, A.; DÍAZ CUÉLLAR, F.; GONZÁLEZ LANDRIÁN, L.; PINEDO MELIS, P.; HERNÁNDEZ GENER, M.E.. Los mapas conceptuales: una poderosa herramienta para el aprendizaje significativo. $A C I M E D$, vol. 15, núm. 5, 1-12, 2007. Disponível em http://scielo.sld.cu/scielo.php?script=sci_arttext\&pid=S1024-94352007000500009\&lng=es\&tlng=es >.

PALAIS DE TOKYO. Interview Celeste Boursier-Mougenot. Disponível em $<$ https://www.youtube.com/watch?v=tN0gw0JgIaQ>. Acesso em 3/02/2020.

PÉREZ, P. La Insurrección Expositiva: cuando el montaje de exposiciones es creativo y divertido. Cuando la exposición se convierte en una herramienta subsersiva. Gijón, España: EdicionesTrea, 2007.

RICO, J. C. Montaje de exposiciones. 3ª reimpr. Madrid: Ediciones Sílex, 2007.

SILVA-CAÑAVERAL, S. J. La práctica artística como lugar de visibilización de las mujeres transgénero de Pereira (Colombia). Revista Estudios Feministas, vol. 27, núm. 3, 1-13, 2019. Doi $<$ https://doi.org/10.1590/1806-9584-2019v27n357219>.

TIRADO DE LA CHICA, A. La exposición de arte desde la transferencia del conocimiento artístico. EARI Educación Artística Revista de Investigación, 10, 168-183, 2019. Doi $<\mathrm{http}$ ://dx.doi.org/10.7203/eari.10.13979>.

TOLEDO CASTELlANOS, R. B. Las Investigaciones Artísticas, Investigaciones de Contexto. Praxis \& Saber, vol. 3, núm. 6,43-88, 2012. Doi <https://doi.org/10.19053/22160159.2003>.

WINCKELMANN, J. J. Historia del arte en la Antiguëdad: seguida de las Observaciones sobre la arquitectura de los antiguos, Madrid: Aguilar, 1763, 1955.

WINCKELMANN, J. J. Reflexiones sobre la imitación del arte griego en la pintura y la escultura, Barcelona: Península, $1^{\mathrm{a}}$ ed. en español, 1987. 
WINCKELMANN, J. J. Lo bello en el arte. Buenos Aires: Nueva Visión, 1964.

ZÚÑIGA SALAS, X. La investigación artística en las artes visuales: estudio de tres casos en la Universidad de Costa Rica. ESCENA. Revista de las artes, vol. 74, núm. 2, 55-73, 2015. Disponível em $<$ https://revistas.ucr.ac.cr/index.php/escena/article/view/21175/21363>. Acesso em 3/02/2020. 